\title{
Die Auslegung der Entsenderichtlinie durch den EuGH in den Entscheidungen Laval und Rüffert*
}

\author{
Juliane Kokott
}

I. Einleitung

II. Schutz der Arbeitnehmerinnen und Arbeitnehmer in der Rechtsprechung des Europäischen Gerichtshofs

III. Auslegung der Entsenderichtlinie durch den Europäischen Gerichtshof

IV. Ausblick

\section{Einleitung}

Die Bedeutung der sozialen Dimension im Recht der Europäischen Union und in der Rechtsprechung des Europäischen Gerichtshofs ist ein aktuelles und wichtiges Thema. Gerade auch im Anschluss an einige Entscheidungen des EuGH wird darüber gegenwärtig vehement diskutiert. Dies überrascht nicht. Gilt es hier doch, die verschiedenen miteinander im Wettstreit stehenden Interessen in einen gerechten Ausgleich zu bringen. Der Einladung zum heutigen Symposium bin ich sehr gerne gefolgt - nicht nur wegen der Bedeutung der aufgeworfenen Fragen, sondern auch weil ich meine, dass sich die Vertreter der Europäischen Institutionen dem Dialog mit der Wissenschaft und Öffentlichkeit stellen sollten.

Mein Amt gebietet mir gleichwohl eine gewisse Zurückhaltung. Zwar war ich an den Verfahren Rüffert und Laval, auf die ich näher eingehen werde, nicht als Generalanwältin beteiligt, doch künftig könnte ich möglicherweise mit ähnlichen Sachverhalten befasst sein. Daher werde ich den Schwerpunkt meiner Ausführungen auf die Darstellung der Grundlinien der Rechtsprechung des Europäischen Gerichtshofs legen und bei der Kommentierung der Urteile Zurückhaltung wahren.

* Meiner Rechtsreferentin Frau Ioanna Dervisopoulos danke ich herzlich für die Unterstützung bei der Erstellung des Manuskripts. 
Grundgedanke der Europäischen Integration ist es, den wirtschaftlichen Fortschritt zu fördern, indem nationale Hürden für den Personen-, sowie Waren-, Dienstleistungsund Kapitalverkehr abgebaut werden und so ein Gemeinsamer Markt geschaffen wird. Von diesem Fortschritt profitieren im Ergebnis alle Bürgerinnen und Bürger in allen Mitgliedstaaten.

Das Funktionieren des Gemeinsamen Marktes bringt jedoch auch Spannungen mit sich. Mit der Stärkung des innergemeinschaftlichen Wettbewerbs, der volkswirtschaftlich für die Union im Ganzen gewinnbringend ist, können für den Einzelnen - zumindest vorübergehend - auch Nachteile verbunden sein. Der vergrößerte Wettbewerbsdruck bringt für Arbeitnehmerinnen und Arbeitnehmer die Gefahr mit sich, geänderte Arbeitsbedingungen akzeptieren zu müssen oder sogar ihre Stelle zu verlieren. Ihre Interessen gilt es daher in besonderem Maße zu schützen.

Auch und gerade vor dem Hintergrund des derzeit noch bestehenden erheblichen Wohlstandsgefälles in der Union stellt es eine bedeutsame Herausforderung für die Fortentwicklung des Europarechts dar, einen gerechten Ausgleich zwischen der Verwirklichung der Grundfreiheiten einerseits und dem Schutz und dem Ausbau sozialer Standards andererseits herbeizuführen. Gemäß Artikel 2 des EU-Vertrages ist die Förderung des wirtschaftlichen und des sozialen Fortschritts eines der grundlegenden Ziele der Union. Ein wesentliches Anliegen des Lissabonner Vertrages ist es zudem, die soziale Dimension der Europäischen Union deutlicher zu betonen.

\section{Schutz der Arbeitnehmerinnen und Arbeitnehmer in der Rechtsprechung des Europäischen Gerichtshofs}

Bevor ich mich dem Gegenstand meines heutigen Vortrags zuwende, möchte ich zunächst die vielfältigen Beiträge in Erinnerung rufen, die der Europäische Gerichtshof in den vergangenen Jahrzehnten zum Schutz der Arbeitnehmerinnen und Arbeitnehmer geleistet hat.

Der Europäische Gerichtshof hat früh erkannt, dass es auf dem Weg zu einem Gemeinsamen Markt der Stärkung sozialer Rechte bedarf. Er hat mit der Betonung sozialer Rechte als unverzichtbarem Bestandteil der Rechtsordnung der Europäischen Union ein Gegengewicht zu den Kräften des Marktes gestärkt und damit die soziale Dimension der Europäischen Union entscheidend mitgestaltet.

$\mathrm{Zu}$ erwähnen ist insofern zunächst die umfassende Rechtsprechung zum Arbeitnehmerschutz in den unterschiedlichsten Bereichen. Beispielhaft möchte ich die Entscheidungen zur Arbeitszeit ${ }^{1}$, zur Urlaubsgewährung ${ }^{2}$, zur befristeten Beschäftigung ${ }^{3}$ und

1 Siehe u.a. Urteile vom 3. Oktober 2000, Simap, C-303/98, Slg. 2000, I-7963; vom 9. September 2003, Jaeger, C-151/02, Slg. 2003, I-8389; vom 5. Oktober 2004, Pfeiffer u.a., C-397/01 bis C-403/01, Slg. 2004, I-8835; und vom 7. September 2006, Kommission/Vereinigtes Königreich, 
zur Teilzeitbeschäftigung ${ }^{4}$ nennen. Oder denken Sie an die Rechtsprechung des Gerichtshofs, mit der er die Gleichbehandlung von männlichen und weiblichen Arbeitnehmern durchgesetzt hat. 5

Auch die Grundzüge der Behandlung entsandter Arbeitnehmerinnen und Arbeitnehmer wurden ursprünglich in der Rechtsprechung des Europäischen Gerichtshofs entwickelt. Danach verwehrt es das Gemeinschaftsrecht den Mitgliedsstaaten grundsätzlich nicht, ihre Rechtsvorschriften oder die von den Sozialpartnern geschlossenen Tarifverträge hinsichtlich der Mindestlöhne auf jedermann zu erstrecken, der einer nichtselbständigen Arbeit in ihrem Hoheitsgebiet nachgeht. ${ }^{6}$ Die Anwendung derartiger Vorschriften muss allerdings dem Schutz der Arbeitnehmer dienen und verhältnismäßig sein. ${ }^{7}$

Diese Rechtsprechung hat Eingang gefunden in den 12. Erwägungsgrund der Entsenderichtlinie ${ }^{8}$, der ich mich nun zuwenden möchte. Bereits der langwierige Gesetzgebungsprozess dieser Richtlinie bestätigt nochmals, dass wir uns hier mit schwierigen Fragen konfrontiert sehen: Die Verwirklichung der Dienstleistungsfreiheit ${ }^{9}$ will in Einklang gebracht werden mit dem Schutz der Arbeitnehmer ${ }^{10}$ und einem fairen Wettbewerb ${ }^{11}$ und somit der Verhinderung von Sozialdumping.

C-484/04, Slg. 2006, I-7471, sowie meine Schlussanträge vom 9. März 2006 in letzterer Rechtssache.

2 Siehe u.a. Urteile vom 26. Juni 2001, BECTU, C-173/99, Slg. 2001, I-4881; vom 6. April 2006, Federatie Nederlandse Vakbeweging, C-124/05, Slg. 2006, I-3423, sowie meine Schlussanträge vom 12. Januar 2006 in letzterer Rechtssache.

3 Siehe u.a. Urteile vom 4. Juli 2006, Adeneler u.a., C-212/04, Slg. 2006, I-6057; und vom 15. April 2008, Impact, C-268/06, noch nicht in der amtlichen Sammlung veröffentlicht, sowie meine Schlussanträge vom 27. Oktober 2005 bzw. vom 9. Januar 2008 in diesen Rechtssachen.

4 Siehe u.a. Urteile vom 13. Juli 1989, Rinner-Kühn, C-171/88, Slg. 1989, 2743; und vom 6. Dezember 2007, Voß, C-300/06, Slg. 2007, I-10573.

5 Siehe u.a. Urteile vom 8. April 1976, Defrenne, 43/75, Slg. 1976, 455; und vom 21. Juni 2007, Jonkman u.a., C-231/06 bis C-233/06, Slg. 2007, I-5149, sowie meine Schlussanträge vom 29. März 2007 in letzterer Rechtssache.

6 Vgl. u.a. Urteile vom 3. Februar 1982, Seco und Desquenne \& Giral, 62/81 und 63/81, Slg. 1982, 223, Rdnr. 14; und vom 24. Januar 2002, Portugaia Construções, C-164/99, Slg. 2002, I-787, Rdnr. 21.

7 Vgl. in diesem Sinne Urteile vom 23. November 1999, Arblade u.a., C-369/96 und C-376/96, Slg. 1999, I-8453, Rdnr. 36, und vom 14. April 2005, Kommission/Deutschland, C-341/02, Slg. 2005, I-2733, Rdnr. 24.

8 Richtlinie 96/71/EG des Europäischen Parlaments und des Rates vom 16. Dezember 1996 über die Entsendung von Arbeitnehmern im Rahmen der Erbringung von Dienstleistungen, AB1. 1997, L 18, S. 1, im Folgenden: Entsende-Richtlinie oder Richtlinie 96/71.

9 Siehe den ersten Erwägungsgrund der Richtlinie 96/71.

10 Siehe den fünften Erwägungsgrund der Richtlinie 96/71.

11 Siehe den fünften Erwägungsgrund der Richtlinie 96/71. 


\section{Auslegung der Entsenderichtlinie durch den Europäischen Gerichtshof}

In der Rechtssache Rüffert hatte das vorlegende Gericht den Gerichtshof gefragt, ob eine Tariftreueklausel wie sie das niedersächsische Vergabegesetz vorsah, eine nicht gerechtfertigte Beschränkung der Dienstleistungsfreiheit darstellt. In der Rechtssache Laval ging es abgesehen von der Streikproblematik um die Frage, ob das schwedische System zur Festlegung der Löhne und weiterer Arbeitsbedingungen im Einklang mit den Vorgaben der Entsenderichtlinie und mit der Dienstleistungsfreiheit steht.

Gemäß Artikel 3 der Entsenderichtlinie sorgen die Mitgliedstaaten dafür, dass Unternehmen den in ihr Hoheitsgebiet entsandten Arbeitnehmern bestimmte Arbeitsbedingungen garantieren, die im Aufnahmemitgliedstaat durch Gesetz oder durch für allgemeinverbindlich erklärte Tarifverträge festgelegt sind. Die Richtlinie zählt dann verschiedene Arbeitsbedingungen auf, z.B. Höchstarbeitszeiten oder Mindestlöhne. Artikel 3 Absatz 7 der Entsenderichtlinie bestimmt, dass die Anwendung von für die Arbeitnehmer günstigeren Beschäftigungs- und Arbeitsbedingungen möglich ist.

Wie ich eingangs bereits betont hatte, war ich als Generalanwältin nicht an den zu besprechenden Fällen beteiligt. In der Rechtssache Rüffert hat mein Kollege Generalanwalt Bot die Schlussanträge vorgelegt. Generalanwalt Bot hatte dafür plädiert, die Entsenderichtlinie so auszulegen, dass sie einem verstärkten nationalen Schutz der entsandten Arbeitnehmer nicht entgegensteht. ${ }^{12}$ Vergleichbar argumentierte Generalanwalt Mengozzi in der Rechtssache Laval. ${ }^{13}$ Auch ich habe eine große Sympathie für dieses Verständnis der Entsenderichtlinie.

Generalanwalt Bot versteht die Richtlinie als ,harten Kern“ von Schutzbestimmungen, die nur als Mindestgarantie zugunsten der entsandten Arbeitnehmer zu verstehen sind. Die Richtlinie habe eine den Mitgliedsstaaten bis dahin zur Verfügung stehende Regelungsmöglichkeit in eine Regelungsverpflichtung umgewandelt. Wie ich bereits dargelegt habe, hatte der Gerichtshof ja schon vor Erlass der Entsende-Richtlinie festgestellt, dass die Mitgliedstaaten unter bestimmten Bedingungen ihre Rechtsvorschriften auch auf entsandte Arbeitnehmer anwenden durften. Aus dieser Erlaubnis machte die Entsende-Richtlinie nach Auffassung des Generalanwalts nun im Hinblick auf bestimmte Mindestbestimmungen zum Schutz der Arbeitnehmer eine Verpflichtung. ${ }^{14}$ Dass die Mitgliedstaaten den entsandten Arbeitnehmern auch einen darüber hinaus gehenden Schutz gewähren können, folgt nach Ansicht des Generalanwalts aus ihrem Artikel 3 Absatz 7.

12 Schlussanträge des Generalanwalts Bot vom 20. September 2006 in der Rechtssache Rüffert, C$346 / 06$, noch nicht in der amtlichen Sammlung veröffentlicht.

13 Schlussanträge des Generalanwalts Mengozzi vom 23. Mai 2007 in der Rechtssache Laval un Partneri, C-341/05, Slg. 2007, I-11767, insbesondere Nrn. 147 und 151.

14 Schlussanträge des Generalanwalts Bot in der Rechtssache Rüffert (zitiert in Fn. 12, Nr. 70). 
Eine Tariftreueklausel stellt daher nach der Ansicht von Generalanwalt Bot einen zulässigen verstärkten nationalen Schutz im Sinne von Artikel 3 Absatz 7 der EntsendeRichtlinie dar. ${ }^{15}$ Eine gegenüber der Richtlinie verstärkte Schutzmaßnahme der Mitgliedstaaten muss allerdings auch mit den Grundfreiheiten vereinbar sein. Dass eine solche Regelung eine Beschränkung des freien Dienstleistungsverkehrs ist, steht nach Ansicht von Bot außer Zweifel. Er sieht diese Beschränkung jedoch aus Gründen des Arbeitnehmerschutzes und zur Verhinderung von Sozialdumping als gerechtfertigt an. ${ }^{16}$ Im Ergebnis hatte Generalanwalt Bot daher dem Gerichtshof vorgeschlagen, die Entsende-Richtlinie und die Dienstleistungsfreiheit dahin auszulegen, dass sie eine Tariftreueklausel erlauben, sofern diese das Verbot der Diskriminierung aus Gründen der Staatsangehörigkeit sowie den Grundsatz der Transparenz beachtet.

Der Gerichtshof ist den Schlussanträgen des Generalanwalts nicht gefolgt. Nach dem Verständnis des Gerichtshofs sieht die Richtlinie ein begrenztes Regularium für den Schutz entsandter Arbeitnehmer vor. Die Richtlinie zähle diejenigen Modalitäten auf, mit denen der nationale Gesetzgeber seine Bestimmungen über Arbeitsbedingungen auf entsandte Arbeitnehmer erstrecken kann. Nach diesem Verständnis kann ein Mindestlohn entweder gesetzlich festgelegt oder aber über den Weg der Allgemeinverbindlichkeitserklärung eines Tarifvertrags bestimmt werden. ${ }^{17}$

Nach Auffassung des Gerichtshofs ermächtigt Artikel 3 Absatz 7 der EntsendeRichtlinie die Mitgliedstaaten nicht, Arbeits- und Beschäftigungsbedingungen durchzusetzen, die nicht in Absatz 1 aufgelistet sind. Nur diese Auslegung der Richtlinie verhindere, dass die Richtlinie leer laufe. Die Aufzählung von bestimmten Bereichen und bestimmten Modalitäten, mit denen Arbeitsbedingungen auf entsandte Arbeitnehmer erstreckt werden könnten, wäre bedeutungslos, wenn über den Absatz 7 auch günstigere Arbeits- und Beschäftigungsbedingungen wie beispielsweise Tariflöhne, die über Mindestlohnsätzen liegen, durch Tariftreueklauseln einseitig durchgesetzt werden könnten. Eine andere Auslegung liefe darauf hinaus, der Entsende-Richtlinie ihre praktische Wirksamkeit zu nehmen. ${ }^{18}$ Ein über Artikel 3 Absatz 1 der Entsende-Richtlinie hinausgehender Schutz scheint nach dem Verständnis des Gerichtshofs somit nur ausnahmsweise nach Artikel 3 Absatz 10 zulässig zu sein, wenn es sich bei ihnen um Vorschriften im Bereich der öffentlichen Ordnung handelt. ${ }^{19}$

Die Entsende-Richtlinie berechtigt daher nach Ansicht des Gerichtshofs einen Mitgliedstaat nicht zum Erlass einer Tariftreueklausel. Im Anschluss hieran prüfte der Ge-

15 Schlussanträge des Generalanwalts Bot in der Rechtssache Rüffert (zitiert in Fn. 12, Nr. 94).

16 Schlussanträge des Generalanwalts Bot in der Rechtssache Rüffert (zitiert in Fn. 12, Nrn. 114ff).

17 Falls die nationale Rechtsordnung kein System zur Allgemeinverbindlicherklärung kennt, können die Mitgliedstaaten gem. Artikel 3 Absatz 8 der Richtlinie 96/71 folgende Mittel wählen: allgemein wirksame Tarifverträge oder repräsentative Tarifverträge.

18 Urteil vom 18. Dezember 2007, Laval un Partneri, C-341/05, Slg. 2007, I-11767; und Urteil vom 3. April 2008, Rüffert, C-346/06, noch nicht in der amtlichen Sammlung veröffentlicht, Rdnr. 33.

19 Urteil Laval un Partneri (zitiert in Fn. 18, Rdnr. 82) und Urteil vom 19. Juni 2008, Kommission/Luxemburg, C-319/06, noch nicht in der amtlichen Sammlung veröffentlicht, Rdnr. 27. 
richtshof, ob diese Auslegung der Richtlinie durch deren Würdigung im Lichte der Dienstleistungsfreiheit bestätigt werde. ${ }^{20}$ Der Gerichtshof prüft die Tariftreueklausel noch einmal an der Grundfreiheit. Zwar bejaht er wie Generalanwalt Bot eine Beschränkung der Dienstleistungsfreiheit. Anders als der Generalanwalt verneint der Gerichtshof aber die Rechtfertigung der Beschränkung durch zwingende Gründe des Allgemeininteresses. Hierbei stützt er sich insbesondere darauf, dass die Tariftreueklausel nur für öffentliche Auftraggeber gelte und nicht auch für private Auftraggeber. ${ }^{21}$ Diese Argumentation ist sicherlich nicht zwingend.

Die Ausführungen des Gerichtshofs in den Urteilen Laval und Rüffert deuteten bereits darauf hin, dass er die in der Entsende-Richtlinie enthaltenen Regelungsmöglichkeiten als abschließend ansieht und darüber hinausgehende Maßnahmen grundsätzlich für unzulässig erachtet. Für den Gerichtshof scheint mir somit die Richtlinie die abschließend kodifizierte Abwägung zwischen Dienstleistungsfreiheit und Arbeitnehmerschutz darzustellen.

In seinem Urteil Kommission/Luxemburg von letzter Woche scheint der Gerichtshof seine Auffassung vom abschließenden Charakter der Entsende-Richtlinie zu verfestigen. ${ }^{22}$ In dieser Rechtssache ging es unter anderem um eine luxemburgische Bestimmung, die die automatische Anpassung der Entlohnung an die Entwicklung der Lebenshaltungskosten auch auf entsandte Arbeitnehmer erstreckte. Die Kommission beanstandete nicht, dass die Mindestlöhne an die Lebenshaltungskosten angepasst werden. Dies sei nach der Entsende-Richtlinie zulässig. Dass die Indexierung aber sämtliche Löhne betreffe, also auch solche, die keine Mindestlöhne sind, verstoße gegen die Richtlinie. Dies hat der Gerichtshof bestätigt. ${ }^{23}$ Eine solche Maßnahme der Indexierung sei in der Entsende-Richtlinie nicht vorgesehen, die abschließend die Aspekte nenne, hinsichtlich derer die Mitgliedstaaten ihre nationalen Bestimmungen auf entsandte Arbeitnehmer erstrecken können. Luxemburg habe auch nicht hinreichend nachgewiesen, dass es sich bei dieser Bestimmung um eine Vorschrift im Bereich der öffentlichen Ordnung im Sinne der Entsende-Richtlinie handele. ${ }^{24}$ In seinem Urteil prüft der Gerichthof die Maßnahme nur mehr an der Richtlinie, anders als bei Rüffert nicht zusätzlich noch am Maßstab der Grundfreiheit.

Welcher Gestaltungsspielraum steht den Mitgliedstaaten nach dem Verständnis des Gerichtshofs für den Schutz entsandter Arbeitnehmer folglich noch zur Verfügung? Unzweifelhaft zulässig sind nach der Rechtsprechung des Gerichtshofs Regelungen in Bezug auf diejenigen Aspekte der Arbeitsbedingungen, die die Richtlinie explizit aufzählt. Bezüglich der Art und Weise wie diese Arbeitsbedingungen auf entsandte Arbeitnehmer erstreckt werden können, stehen den Mitgliedstaaten zwei Optionen zur Verfü-

20 Urteil Rüffert (zitiert in Fn. 18, Rdnr. 36ff).

21 Urteil Rüffert (zitiert in Fn. 18, Rdnr. 40).

22 Urteil Kommission/Luxemburg (zitiert in Fn. 19, Rdnr. 26).

23 Urteil Kommission/Luxemburg (zitiert in Fn. 19, Rdnr. 47).

24 Urteil Kommission/Luxemburg (zitiert in Fn. 19, Rdnr. 54). 
gung: die gesetzliche Festlegung oder ein für allgemeinverbindlich erklärter Tarifvertrag. 25

Für die entsendenden Unternehmen hat die Auslegung des Gerichtshofs sicher den Vorteil einer größeren Rechtssicherheit und Vorhersehbarkeit der Löhne, die bei Dienstleistungen in einem anderen Mitgliedstaat zu entrichten sind. Gleichwohl besteht die Gefahr einer Entwicklung der Löhne im Aufnahmemitgliedstaat in Richtung auf die Mindestgewährleistungen.

\section{Ausblick}

Die Urteile des Gerichtshofs Viking ${ }^{26}$, Laval und Rüffert werden gegenwärtig stark kritisiert. Auch meine Vorredner haben heute verschiedene Bedenken formuliert. Bereits die Schlussanträge der Generalanwälte Bot und Mengozzi zeigen, dass auch innerhalb des Gerichtshofs kein einhelliges Meinungsbild herrscht.

Ich möchte in diesem Zusammenhang vor allem einen Gesichtspunkt betonen, der mir von vielen kritischen Stimmen, die etwa durch das Urteil Laval das Ende des Streikrechts eingeläutet sehen, nicht hinreichend berücksichtigt wird. Man sollte sich bewusst sein, dass der Gerichtshof in den betreffenden Urteilen nur über die jeweilige spezifische Vorlagefrage entschieden hat. Deren Ausgangspunkt sind jeweils ganz konkrete, durch zahlreiche Besonderheiten gekennzeichnete Rechtsstreite. Eine genaue Lektüre der Urteile zeigt, dass der Gerichtshof gerade diese Besonderheiten hervorhebt und an ihnen sein Urteil festmacht. So betonte er im Urteil Laval den nationalen Kontext, in den sich die kollektiven Maßnahmen einfügten. Für diesen spezifischen Kontext war kennzeichnend, dass es keine Vorschriften gab, die hinreichend genau und zugänglich waren, um einem Unternehmen zu ermöglichen, festzustellen, welche Verpflichtung es hinsichtlich des Mindestlohns beachten muss. ${ }^{27}$

Der Gerichtshof hat keine allgemeine Aussage zum Verhältnis von Grundfreiheiten und Grundrechten getroffen. Insbesondere hat er in den aktuellen Urteilen nicht entschieden, dass das Streikrecht oder gar die Menschenwürde grundsätzlich hinter den Grundfreiheiten zurückzustehen hat. Grundfreiheiten und Gemeinschaftsgrundrechte stehen vielmehr auf einer Ebene und müssen miteinander in Einklang gebracht werden.

Indem der Gerichtshof besonders die spezifischen Charakteristika der Fälle betont, signalisiert er meines Erachtens unmissverständlich, anhand der Besonderheiten künftiger Vorlageverfahren seine Rechtsprechung weiter ausdifferenzieren zu wollen.

25 Falls das nationale Rechtssystem keine Allgemeinverbindlicherklärung kennt, können die Mitgliedstaaten gem. Artikel 3 Absatz 8 der Richtlinie 96/71 folgende Mittel wählen: allgemein wirksame Tarifverträge oder repräsentative Tarifverträge.

26 Urteil vom 11. Dezember 2007, International Transport Workers' Federation und Finnish Seamen's Union (,Viking Line“), C-438/05, noch nicht in der amtlichen Sammlung veröffentlicht.

27 Urteil Laval un Partneri (zitiert in Fn. 18, Rdnr. 110). 
Lassen Sie mich zum Abschluss betonen: Bei aller Kritik an den konkreten Urteilen darf nicht übersehen werden, dass der Gerichtshof darin im Grundsatz aus sozialer Perspektive auch positiv zu bewertende Ausführungen macht: Er erkennt das Streikrecht als Grundrecht an. Und bemerkenswerterweise greift er im Urteil Laval den Begriff des Sozialdumpings auf und sieht im Streikrecht zum Schutz der Arbeitnehmer gegen Sozialdumping ein zwingendes Allgemeininteresse. ${ }^{28}$

Ich komme zum Ende meiner Ausführungen. Meines Erachtens läuft insbesondere die öffentliche Diskussion Gefahr, die Entscheidungen Rüffert und Laval überzubewerten. Beide Entscheidungen sind kein Wendepunkt am Ende einer langen Kette von Entscheidungen des Gerichtshofs, die sich im Spannungsfeld von Grundfreiheiten und sozialen Rechten bewegen. Wie sicherlich auch dieses Symposium zeigen wird, ist vielmehr festzuhalten, dass diese Entscheidungen Pendelausschläge der Rechtsprechung des Europäischen Gerichtshofs darstellen, in dem Bemühen, in einer sich rasch entwickelnden Union die dargestellten schwierigen Abwägungsfragen auszudifferenzieren und von Fall zu Fall weitere Aspekte zu berücksichtigen.

Soviel ist sicher: Der Gerichtshof wird seine Rechtsprechung noch weiter entwickeln und präzisieren. Auch der Diskurs in der Fachöffentlichkeit und Diskussionsveranstaltungen wie die heutige leisten insofern einen wichtigen Beitrag. Dass die heutige Veranstaltung die Möglichkeit eröffnet, nicht lediglich die deutsche Perspektive, sondern dank zahlreicher Teilnehmer aus anderen Mitgliedstaaten auch deren Sichtweise kennen zu lernen, stellt eine wertvolle Chance dar.

28 Wörtlich heißt es im Urteil Laval un Partneri (zitiert in Fn. 18) in Randnr. 103: „... dass im Recht auf Durchführung einer kollektiven Maßnahme, die den Schutz der Arbeitnehmer des Aufnahmemitgliedstaates gegen ein etwaiges Sozialdumping zum Ziel hat, ein zwingender Grund des Allgemeininteresses“ liegen kann. Siehe hierzu bislang nur Urteil vom 19. Januar 2006, Kommission/Deutschland, C-244/04, Slg. 2006, I-885, Rdnr. 61. 